Handbuch der Zoologie

Handbook of Zoology

Band/Volume VIII Mammalia

Karl F. Koopman

Chiroptera: Systematics

Teilband/Part 60 


\section{Handbuch der Zoologie}

Eine Naturgeschichte der Stämme des Tierreiches

\section{Handbook of Zoology}

A Natural History of the Phyla of the Animal Kingdom

Gegründet von / Founded by Willy Kükenthal

Fortgeführt von / Continued by M. Beier, M. Fischer, J.-G. Helmcke,

D. Starck, H. Wermuth

\section{Band/Volume VIII Mammalia Teilband/Part 60}

Herausgeber/Editors J. Niethammer, H. Schliemann, D. Starck

Schriftleiter/Managing Editor H. Wermuth

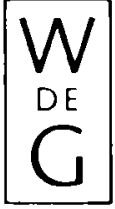

Walter de Gruyter · Berlin · New York 1994 
Karl F. Koopman

\section{Chiroptera: Systematics}

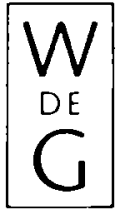

Walter de Gruyter · Berlin • New York 1994 
Autor/Authors

Dr. Karl F. Koopman

American Museum of Natural History

Central Park West at 79th Street

New York, New York 10024-5192

U.S.A

Schriftleiter/Managing Editor

Dr. Heinz Wermuth

Falkenweg 1

D-71691 Freiberg

Federal Republic of Germany

\section{Verlag/Publisher}

Walter de Gruyter \& Co.

Genthiner Straße 13

D-10785 Berlin

Federal Republic of Germany

Tel. (030) $26005-0$

Telefax (030) 26005-2 51
Walter de Gruyter, Inc. 200 Saw Mill River Road Hawthorne, N.Y. 10532

U.S.A

Telefax (914) 747-1326
Tel. (914) 747-0110

Das Buch enthält 192 Abbildungen.

With 192 illustrations.

(0) Printed on acid-free paper which falls within the guidelines of the ANSI to ensure permanence and durability.

Die Deutsche Bibliothek - CIP-Einheitsaufnahme

Handbuch der Zoologie - eine Naturgeschichte der Stämme des

Tierreiches / gegr. von Willy Kükenthal. Fortgef. von M. Beier

... - Berlin ; New York : de Gruyter.

Teilw. mit Parallelt.: Handbook of zoology

NE: Kükenthal, Willy [Begr.]; Beier, Max [Hrsg.]; PT

Bd. 8. Mammalia / Hrsg. J. Niethammer ...

Teilbd. 60. Chiroptera : systematics / Karl F. Koopman. - 1994

ISBN 3-11-014081-0

NE: Niethammer, Jochen [Hrsg.]; Koopman, Karl F.

Library of Congress Cataloging-in-Publication Data

\footnotetext{
Handbuch der Zoologie

Parallel title: Handbook of zoology.

Vol. 8, part 56 has: fortgeführt von M. Beier, ... - [et al.].

Issued in parts.

German on English.

Includes bibliographies and indexes.

Contents: v. 1. Ed. Protozoa. Porifera. Coelenterata.

Mesozoa - v. 3. Bd. 1. Hälfte. Tardigrada. Pentastomida.

Myzostomida. Arthropoda: Allgemeines. Crustacea. 2.

Hälfte. Chelicerata, Pantopoda, Onychophora, Vermes

Oligomera. (2 v.) - [etc.] - v. 8. Bd. Mammalia.

1. Zoology. I. Kükenthal, W. G. (Willy Georg),

1861-1922. II. Krumbach, Thilo, b. 1874.

III. Handbook of zoology.

QL45.H2

591

$23-1436$

ISBN 3110140810
}

Herausgeber/Editors

Professor Professor

Dr. Jochen Niethammer Dr, Harald Schliemann

$\begin{array}{ll}\text { Zoologisches Institut der } & \text { Zoologisches Institut und } \\ \text { Universität Bonn } & \text { Zoologisches Museum }\end{array}$

Universität Bonn

Poppelsdorfer Schloß

Martin-Luther-King-Platz 3

D-20146 Hamburg

Federal Republic of Germany Federal Republic of Germany

Professor

Dr. med. Dr, phil. h.c.

Dietrich Starck

Balduinstraße 88

D-60599 Frankfurt

Federal Republic of Germany 